\title{
PULMONARY OEDEMA IN ACUTE GLOMERULONEPHRITIS
}

BY

\author{
J. G. WILSON* \\ From the Adelaide Children's Hospital, Adelaide, South Australia
}

(RECEIVED FOR PUBLICATION APRIL 4, 1961)

Pulmonary oedema in association with acute glomerulonephritis is uncommon (Wood, 1956; Nelson, 1959), and the pathogenesis is as yet far from clear. De Wardener (1958) states that $80 \%$ of the cases of acute nephritis recover completely and $5 \%$ die in one or two weeks of either acute cardiac failure, acute renal failure or hypertensive encephalopathy. The remaining $15 \%$ subsequently develop chronic nephritis. Cases dying in acute cardiac failure, therefore, are rare. Whether the pulmonary oedema in these cases is due to acute cardiac failure or not will be discussed later.

This paper gives an account of three children who suddenly developed the clinical features of acute pulmonary oedema. A discussion of the pathogenesis with special reference to renal diseases is given.

\section{Case Reports}

Case 1. A girl aged $11 \frac{1}{2}$ years, the eldest child of an Italian family with three other children, was admitted on October 10, 1959.

Family History. The three younger children were well, apart from recent colds. They had attended their doctor frequently for upper respiratory tract infections and otitis media. Two other children had died in Italy before immigration, one, when 9 days old, from congenital abnormalities and the other from meningitis when 2 months old. The father had been in bed with 'influenza' during the past week, otherwise he had always been well. There were no other family illnesses.

Previous Illnesses. She had always been a healthy robust girl and had never really been ill before. She had had measles and mumps, but had not had any immunizing injections.

Present Illness. For one week before admission to hospital she had had a cold and a discharging ear. Three days before admission she still had a discharging ear, temperature $102^{\circ} \mathrm{F}$., and her doctor was called. $\mathrm{He}$ diagnosed acute tonsillitis and gave a penicillin injection, 900,000 units. The next day she was a little

\footnotetext{
* Present address: 33 Statenborough Street, Leabrook, South
} Australia. better. The temperature had fallen to $99^{\circ} \mathrm{F}$. and she was given another injection of penicillin. On the morning of the following day she complained of abdominal pain centred mainly over the right iliac fossa. The temperature was $98^{\circ} \mathrm{F}$. and pulse rate 90 per minute. The child became somewhat hysterical at the thought of another injection, so this was not given.

Because of the abdominal pain, her doctor called again at $8 \mathrm{p} . \mathrm{m}$. when the abdomen was normal, but he noticed a very slight grunt in her breathing. The rate was slow and she was sleeping comfortably. The mother stated she had a fleeting rash since the last injection. During the evening she became progressively more short of breath, developed a cough and had to sit up in bed. The window was opened to help her to get more air. By 4 o'clock the next morning she became so distressed she was brought to the hospital.

On admission her temperature was $99^{\circ} \mathrm{F}$., pulse 128 per minute; she was very dyspnoeic, was sitting up, and was restless and cyanotic. She was a rather plump Italian girl with no peripheral or facial oedema. She had a discharging right ear and the fauces were red. Trachea was midline. Heart rate was rapid with normal findings on auscultation. In the lungs there were scattered râles mainly at the bases and a reduced air entry at the left base. Blood pressure was $140 / 90 \mathrm{~mm}$. Hg. No other significant abnormality was found. The urine was not tested owing to the difficulty of collecting a specimen. A provisional diagnosis of a left-sided pneumonia was made and she was treated accordingly with oxygen, intramuscular chloramphenicol $500 \mathrm{mg}$. statim then $200 \mathrm{mg}$. six-hourly, intravenous aminophyllin $240 \mathrm{mg}$. and paraldehyde $2 \mathrm{ml}$. intramuscularly to help control her restlessness. A throat swab showed Streptococcus viridans only. Chest radiographs of both lung fields showed widespread interstitial pulmonary oedema and the cardiothoracic ratio was increased (Fig. 1).

From admission at 4 a.m. on October 19, 1959, to death at $2.35 \mathrm{p} . \mathrm{m}$. the next afternoon her course was progressively downhill. She improved temporarily with oxygen, but later became more dyspnoeic, restless and cyanosed. At 12 noon her condition worsened and much blood-stained frothy mucus was coughed up from her mouth. Intravenous injections of digoxin $0.5 \mathrm{mg}$. and hydrocortisone $50 \mathrm{mg}$. were given at this stage. 


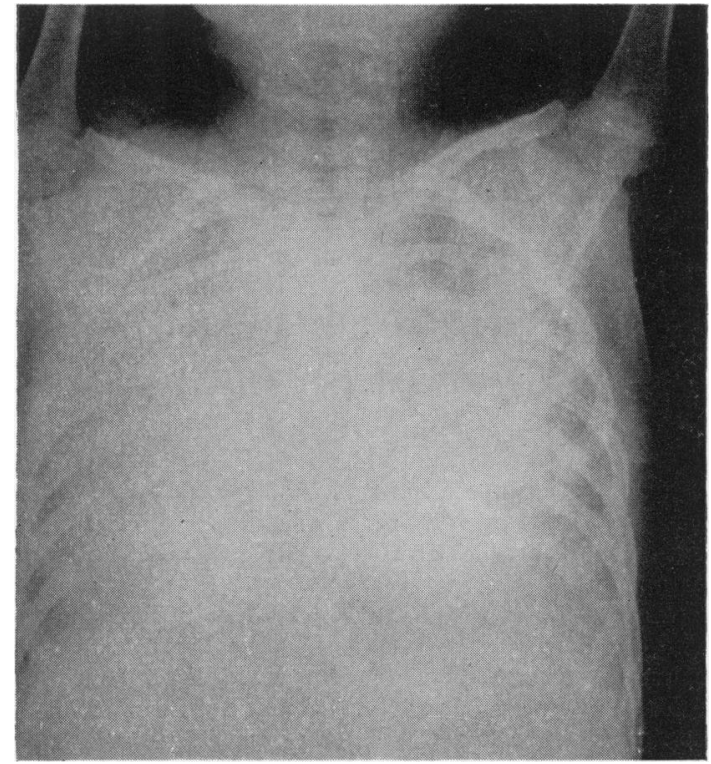

Fig. 1.-Chest radiograph: both lung fields show widespread interstitial pulmonary oedema; the cardiothoracic ratio is probably increased.

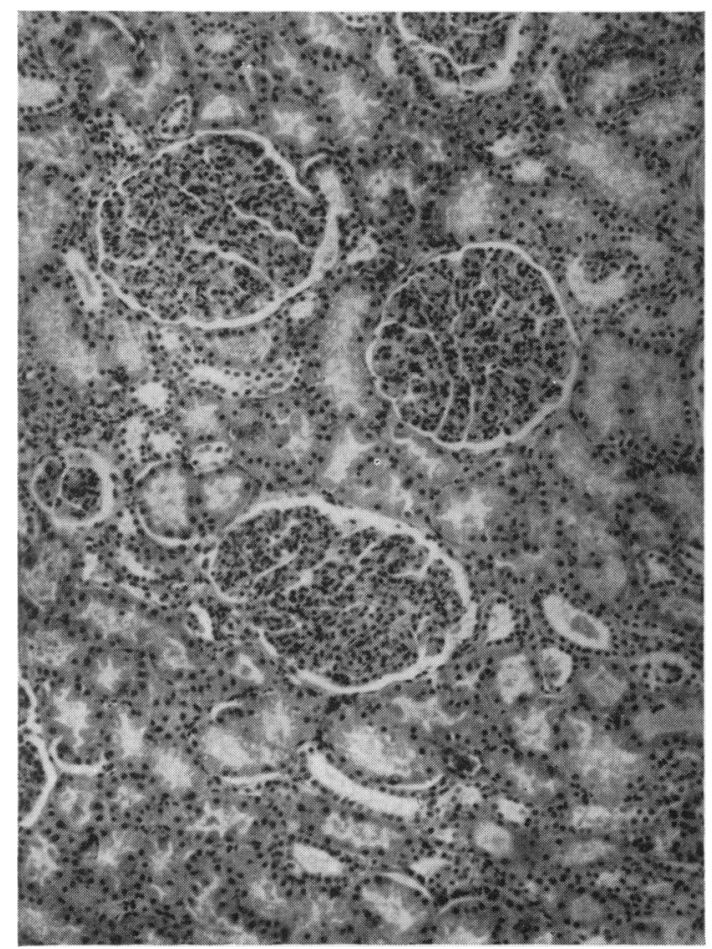

FIG. 2.-The glomerular tufts of the kidney are enlarged, hypercellular and vascular. (H. and E. $\times 85$.)
The salmon pink frothy mucus increased in amount and obstructed her airway, and in spite of attempts to clear this she died at 2.35 p.m.

\section{Autopsy. (PM 121/59 A.C.H.)}

Pleural Cavities. Each contained approximately $350 \mathrm{ml}$. of clear yellow fluid. Trachea contained a moderate amount of watery fluid streaked with fibrinous threads. Lungs were dull purple and heavy, and on incision airless and oedematous. Pericardium contained approximately $75 \mathrm{ml}$. of clear straw-coloured fluid. Heart weight was $170 \mathrm{~g}$. (normal is $124 \mathrm{~g}$.). The left ventricle was moderately hypertrophied and the right ventricle dilated. Kidneys: the weight of the right was $99 \mathrm{~g}$.; left, $94 \mathrm{~g}$. On incision the cortex was increased in depth and pale with fine red streaks and very small white glistening glomeruli projecting from the cut surface. The medulla was deeply congested. A blood urea nitrogen performed on post-mortem blood was $22 \mathrm{mg} . / 100 \mathrm{ml}$.

MiCROSCOPIC EXAMINATION. In the kidney glomeruli (Fig. 2) the tufts were comparatively avascular and hypercellular and infiltrated with small numbers of polymorphonuclear leucocytes. Some showed an exudate of red blood cells in the capsular space. A few tubules contained red blood cells while others contained an eosinophilic amorphous material. The capsular epithelium showed no crescent formation, nor was there fibrosis of any glomeruli. The interlobular arterioles and afferent arterioles were normal and there was no infiltrate in the interstitial tissues. Lung alveoli were expanded and the walls slightly thickened and congested. Many alveoli contained a finely granular and sometimes fibrillar exudate which in places showed a tendency to condense against the alveolar wall, forming a membrane. In addition there was a cellular exudate composed principally of red blood cells with a few polymorphonuclear leucocytes and macrophages. No eosinophils were seen.

In summary the autopsy revealed bilateral pleural effusion; pulmonary oedema; cardiac hypertrophy, and kidneys with prominent hypercellular avascular glomeruli. The immediate cause of death was pulmonary oedema; the primary cause, acute nephritis.

Comment. Unfortunately no urine was available at autopsy for examination. However, the diagnosis of acute nephritis is certain pathologically, although unfortunately not suspected clinically.

Case 2. A girl aged 5 years and 9 months, was admitted to hospital on April 24, 1960.

History. She developed a cold two days before admission and the next day she began to vomit, but was not ill. On the morning of admission she continued to vomit and became short of breath. She was given 900,000 units of penicillin with no apparent improvement. This shortness of breath became progressively worse throughout the day and by 6.25 p.m. she was admitted to the hospital in a state of severe pulmonary oedema. 
Previous Illnesses. She was alleged to have had vomiting episodes previously, sometimes associated with shortness of breath. The real nature of these attacks is unknown, but they were never severe nor incapacitating and certainly never as severe as the present episode.

Family History. Two aunts had had rheumatic fever and there was asthma in her mother's family.

Examination revealed a restless child, grossly dyspnoeic and cyanosed with cold extremities; temperature $99^{\circ} \mathrm{F}$.; examination of heart showed apex beat in the fifth intercostal space just outside the mid-clavicular line. Heart sounds were rapid with a gallop rhythm. The blood pressure was $120 / 90 \mathrm{~mm}$. $\mathrm{Hg}$ in spite of the collapsed state of the child. Pulsus alternans was noted. The radial pulses had poor volume and the jugular venous pressure was not elevated. No hepatomegaly or peripheral oedema were noted. Coarse bubbling râles were found throughout both lung fields. Chest radiograph showed an enlarged heart with widespread pulmonary opacities.

Intravenous cannulation was instituted to facilitate intravenous therapy. Vigorous attempts at resuscitation were made with the administration of digoxin, aminophyllin, hydrocortisone, erythrocin, chloramphenicol, mersalyl and paraldehyde. Oxygen was administered continuously. From admission until death, five and a half hours later, there was a steady deterioration. Morphine sulphate gr. 1/6 was given intravenously in an attempt to control the pulmonary oedema, but with little effect. Vigorous attempts were made to clear her airway with intubation and suction, and positive pressure ventilation was attempted in an endeavour to improve oxygenation and control the oedema. Her upper airway was cleared temporarily only and she steadily became more anoxic. She became unconscious with bloodstained frothy fluid pouring out of the upper respiratory passages and died.

Unfortunately, it was not possible, owing to her desperate condition, to collect any urine, but in view of the similarity to the first case, the diagnosis of acute nephritis was suspected.

Autopsy. (PM 26/60 A.C.H.)

Pleural Cavities. Each contained approximately $40 \mathrm{ml}$. of clear yellow fluid. Pericardial cavity contained $35 \mathrm{ml}$. of clear yellow fluid. There was frothy bloodstained mucus in the upper respiratory tract, trachea and bronchi. The tracheobronchial lymph nodes were enlarged. Both lungs were heavy and purplish. On incision all lobes were fleshy with blood-stained watery fluid pouring freely from the cut surfaces. Right-sided chambers of the heart were dilated. The ventricular wall was hypertrophied and measured $14 \mathrm{~mm}$. in thickness. In each kidney the capsules stripped easily; the cortex was pale, medullary rays were not visible. The pyramids were dark and congested and the pelves normal.

Biochemical Estimations. Blood urea nitrogen: $60 \mathrm{mg} . / 100 \mathrm{ml}$.; blood sugar: $80 \mathrm{mg}$. $/ 100 \mathrm{ml}$.; urine contained albumin +; a blood culture was negative.

Microscopic Examination. Scattered throughout the lungs were focal areas of bronchiolitis (Figs. 3 and 4).

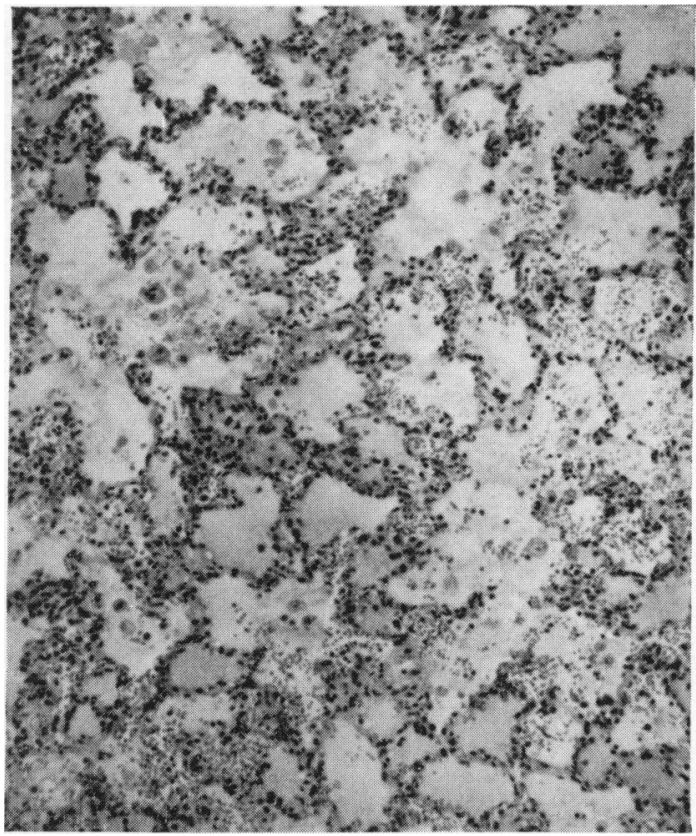

Fig. 3.- $($ H. and E. $\times 85$. $)$

Both are sections of the lung, Fig. 4 being at a higher power than Fig. 3. The alveoli are filled with oedema fluid containing red blood cells and a few macrophages.

FIG. 4.-(H. and E. $\times$ 296.)

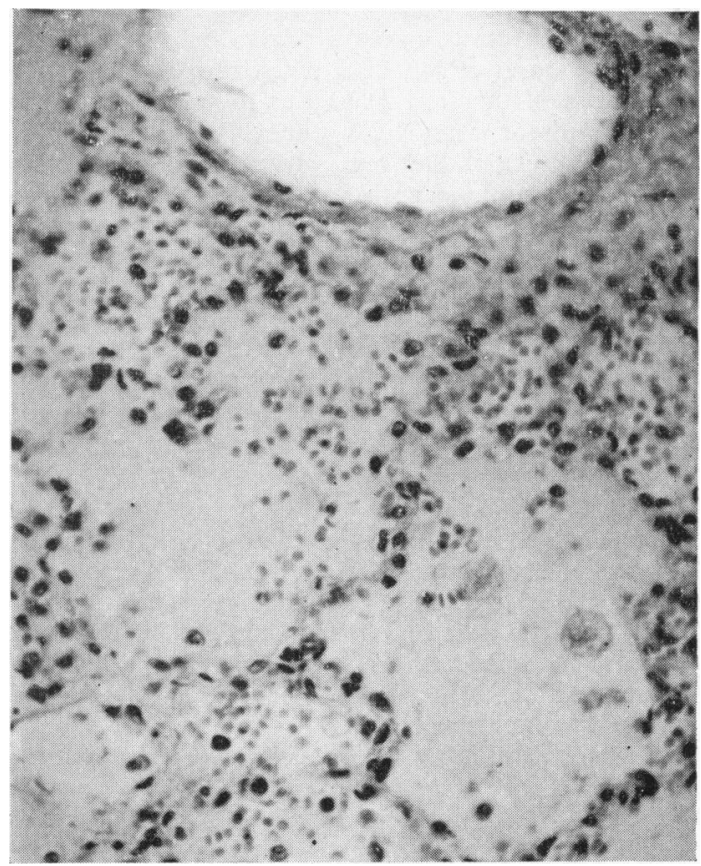


Other areas showed pulmonary oedema and intraalveolar haemorrhages. The alveolar walls were congested. Muscular hypertrophy and mild fatty changes were found in the heart. There were diffuse glomerular changes which included avascularity, cellular proliferation, leucocytic infiltration and 'hyaline thrombi' formation in the kidneys. There were no changes in the tubules, vessels or interstitial tissue.

In summary the autopsy showed a female child with acute pulmonary oedema, congestive cardiac failure, hypertrophied left ventricle and diffuse glomerular changes.

The primary cause of death was acute nephritis; the immediate cause, pulmonary oedema.

Comment. Throat swabs were collected from other members of the family and in one brother haemolytic streptococci were cultured and he was subsequently treated for this.

The intravenous morphia in this case did not seem to help the child from left ventricular failure, a fact which is unusual in pulmonary oedema. She became more comatose and did not regain consciousness after the injection. There was no elevation of the jugular venous pressure noted in this child. Possibly she was in extremis before she was examined and this sign, which is always difficult to assess in children, was not present. The blood pressure was $120 / 90 \mathrm{~mm}$. $\mathrm{Hg}$ at a time when the child was desperately ill and in peripheral circulatory failure with a virtually absent radial pulse. This may suggest that the pressure had been previously higher, but we do not know.

These two cases show many points of similarity and demonstrate several interesting features. In both cases there was a history of an upper respiratory tract infection before the onset of the fatal pulmonary oedema. The significance of this is uncertain as in only one member of the family of Case 2 was a haemolytic streptococcus found. Both cases had been given penicillin injections before admission and both were alleged to have been in good health before the onset of this illness. It would have been valuable to have access to a recent medical examination or a urine test to be certain of this point. Abdominal pain was a feature of Case 1, whereas vomiting attacks had occurred in Case 2. The cause of the abdominal pain and vomiting episodes must remain uncertain. However, it is not impossible that these attacks represented minor episodes of pulmonary oedema, possibly with pleural involvement giving rise to pain.

The degree of hypertension was minimal in both cases. However, the presence of a recordable blood pressure in Case 2 in spite of other evidence of circulatory failure did suggest the possibility of antecedent hypertension. Certainly in each case the hypertension alone was insufficient to lead to cardiac failure and pulmonary oedema (Wood, 1956).

The diagnostic difficulties must also be stressed. In each case in spite of a temperature of $99^{\circ} \mathrm{F}$. a diagnosis of a severe bronchopneumonia was made. There were no clinical features to suggest acute nephritis nor other evidence of cardiac failure. Hypertension was not a feature of either case and we had no urine findings to assist in the diagnosis. The chest radiograph was most helpful in each case and showed the features of pulmonary oedema before the final stage of coughing up salmon-coloured sputum occurred. Thus early diagnosis of this condition is difficult and it seems that once the pulmonary oedema is established no treatment at present available is of any value.

Case 3 represents another recent case of nephritis and pulmonary oedema in whom the features of cardiac failure were more definite.

Case 3. The patient was a male aged 1 year and 10 months, who, four days after a supposed attack of bronchopneumonia and otitis media, complained of abdominal pain. He was treated with penicillin for his pneumonia which resolved rapidly and he appeared quite well until the onset of the upper abdominal pain. On admission to hospital he was slightly cyanosed with grunting respirations. Temperature was normal. There were coarse râles over both lung fields. The liver was palpable two fingers below the right costal margin and the jugular venous pressure was raised. A chest radiograph revealed an enlarged heart with small bilateral pleural effusions. In spite of therapy he died 24 hours after admission.

Autopsy. (PM 58/57 A.C.H.) The heart was grossly hypertrophied; the lungs were a dull violet colour, firm and did not collapse; and there was much frothy fluid exudate from the cut surfaces. Moderate enlargement of the hilar lymph nodes was noted. Much frothy fluid also was found in the trachea and main bronchi. The cut surfaces of the kidneys were pale.

Histological Examination. There was congestion of the capillaries of both lungs and much oedema of the alveoli with lamination of a precipitated protein-containing exudate. Polymorphonuclear leucocytes were found in focal patches in the alveolar spaces.

The heart muscle showed hypertrophy of the muscle fibres of both ventricles.

The glomeruli in the kidneys were large, bloodless and hypercellular. Some showed an increase in eosinophilic material with a few polymorphs. There was an occasional cast containing polymorphs in the tubules with some hyaline droplet formation in the tubular epithelial cells.

In summary: acute nephritis with hypertrophy and dilatation of the heart with oedema of the lungs. The cause of death was thought to be due to cardiac failure occurring during the course of an acute glomerulonephritis.

Comment. This case, both clinically and pathologically, appears similar to the previous two cases. However, the features of cardiac failure with a grossly hypertrophied and dilated heart were more obvious. The jugular venous pressure was elevated. As in the first two cases, there were no urine findings, a respiratory tract infection appeared to be the precipitating cause of 
the nephritis and penicillin was the drug used to treat the pneumonia. In retrospect the pneumonia could have been a mild episode of pulmonary oedema possibly complicated by some infection. There were, however, a few leucocytes in the alveolar spaces.

\section{Pathogenesis of Pulmonary Oedema}

In a discussion on the pathogenesis of pulmonary oedema it is convenient to consider a number of causes (Barach, Martin and Eckman, 1938).

(1) Left ventricular heart failure;

(2) Increased capillary permeability;

(3) Alteration in pressures within the lung:

(a) a persistently high intrathoracic negative pressure

(b) an abrupt termination of backward pressure against the pulmonary capillaries.

In such conditions as tracheal stenosis the heightened intrathoracic negative pressure may encourage pulmonary oedema. Increased blood flow through the lungs under these conditions and possibly some degree of anoxic damage to the alveolar epithelium may increase capillary permeability and so lead to further pulmonary oedema. Sometimes with the performance of a tracheotomy the sudden change of pressure within the chest may lead to pulmonary oedema. However, the two common causes are left ventricular heart failure and increased capillary permeability from a multitude of causes.

Pulmonary oedema has been reported as occurring in many conditions, and in each an explanation is difficult to find. The occurrence of pulmonary oedema during salicylate therapy was reported by Reid, Watson and Sproull (1950) and a further case was reported by Sutcliffe (1955). More recently Granville-Grossman and Sergeant (1960) reported cases of pulmonary oedema due to salicylate intoxication. In these cases the explanation appeared to be the hypernatraemia that arises in conjunction with over-hydration during treatment. However, the comparative rarity of the condition, the low dosage of salicylate sometimes used and the absence of signs of salicylate intoxication suggest a sensitivity reaction to salicylate in some cases. Acute pulmonary oedema has been seen in cases of hypoglycaemic coma (Weber and Blum, 1942), epileptic fits (Ohlmacher, 1910) and numerous other central nervous system conditions. Cerebral wounds seen during World War I in soldiers were occasionally complicated by pulmonary oedema (Moutier, 1918). The Arnold-Chiari malformation has terminated in pulmonary oedema. Experimental pharmacology has favoured the vagus nerve as a cause of these effects. Stimulation of the efferent path of the vagus leads to vasodilatation of the lung capillaries and congestion with ultimate transudation of fluid into the alveoli. The suggestion is that cerebral oedema following hypoglycaemic coma, epileptic fits, etc. leads to irritation of the vagus nerve with consequent pulmonary oedema (Weber and Blum, 1942).

Many explanations have been given for the pulmonary lesions in acute rheumatism (Hadfield, 1938; Brit. med. J., 1955). The signs of left lower lobar consolidation previously ascribed to a spreading rheumatic process are now known to be due to lobar compression by pericardial effusion. The abrupt pleurisy sometimes seen is thought to be due usually to pulmonary infarction. It is felt now that cardiac failure is the reason for the congestive changes seen in the lung in acute rheumatic fever. There is, however, a group in which this does not apply, and in a few of these, pulmonary oedema due to salicylates may be the explanation. Similar lesions to those seen in rheumatic fever have been demonstrated in a sulphonamide sensitivity (French, 1946), and still others have been produced experimentally in rabbits sensitized to egg-white protein (Cannon, Walsh and Marshall, 1941). Undoubtedly, pulmonary oedema can be produced by these sensitizing reactions.

Pulmonary oedema to a greater or lesser degree is comparatively common in pneumonia. In these cases an increase in capillary permeability as a result of the infection may be the cause. When the oedema is the main pathological feature these cases may be indistinguishable clinically from the three cases described when they present suddenly, and rapidly become asphyxiated and die. We have recently seen two such cases in girls in our hospital. In neither case were the lesions of acute nephritis to be seen in the kidney.

\section{Pulmonary Oedema in Relation to Renal Disease}

The first report in Australian literature of pulmonary oedema in association with renal disease was given by White (1907), who described the clinical record of a case of acute suffocative pulmonary oedema in a male of 25 years, who had had several episodes of pulmonary oedema associated with hypertension and cardiac failure. $\mathrm{He}$ also had the clinical features of nephritis with some oedema and albumin in the urine. This case was undoubtedly one of congestive cardiac failure with a large heart, triple rhythm and hypertension. Both morphine and venesection improved the patient on several occasions.

Stanton and Tange (1958) have described nine cases of the combination of pulmonary haemorrhage 
and glomerulonephritis. They mention 12 other cases from the literature, but suggest that this does not, in all probability, reflect its real frequency. They suggest the term Goodpasture's syndrome for the condition of glomerulonephritis complicated by severe idiopathic pulmonary haemorrhage. Goodpasture (1919) described a case during the influenza epidzmic of that year with this combination of lesions and felt that they were an unusual manifestation of influenza. Stanton and Tange's cases are a little different from ours in that they persisted with hacmoptyses for several weeks or months before the final episode overtook the patients. The final episode, however, was of sudden onset with breathlessness and features of pulmonary oedema very similar to our cases. These authors state that the most acute cases of this syndrome may be clinically indistinguishable from cases of glomerulonephritis complicated by severe bronchopneumonia. They mention two cases aged 25 years and 8 years who died suddenly and in whom a chest radiograph showed bilateral diffuse pulmonary lesions; at autopsy the lungs showed oedema fluid with red cells in the alveoli, but also many polymorphs as well. Our cases showed a few polymorphs in the alveolar exudate similar to these two cases and it is difficult to be certain what part infection played in the illness of these patients. Another feature of th:ir nine cases was the fact that the average age was 25 years. It appears, therefore, to be a disease of young adults.

More recently from Manchester a report of 65 cases of acute nephritis in whom 37 showed radiologically detectable lung lesions was given by Holzel and Fawcitt (1960). The majority fell into the school age and the authors stated 'It seems puzzling, however, that the typical picture of pulmonary oedema was seen in only two out of 37 cases with pulmonary lesions.' The other 35 cases showed partial collapse, consolidation, interlobar and pleural effusions. All were seen at the onset of the illness in the hydraemic stage, and spontaneous and rapid resolution occurred after the onset of the diuresis. These facts prompted the authors to postulate that these lung lesions were due to the accumulation of oedema fluid itself and were probably not inflammatory in origin nor due to cardiac failure. The reason given for the accumulation of the oedema fluid, however, was not entirely convincing.

\section{Theories of Pathogenesis in Relation to Our Cases}

In the three cases described the features of pulmonary oedema were found in association with lesions in the kidney glomeruli such as are found in acute glomerulonephritis. It is reasonable, therefore, to assume that acute nephritis should be the primary diagnosis and that both the lung and kidney lesions should be part of the same disease process. The mechanism of the changes in the lungs is difficult to explain. The mechanism causing the pulmonary oedema in these cases is said to be due to acute left ventricular heart failure (Wood, 1956). The onset in these cases was extraordinarily rapid, and in the absence of other signs of heart failure either clinically or at autopsy, this explanation seems unlikely. However, the presence of left ventricular hypertrophy at autopsy in each of two cases is an interesting feature and needs explanation. Hypertrophy of cardiac muscle suggests that the disease process may not have been as sudden as would appear clinically. Unfortunately, we know nothing of the children before the terminal illness, so it is possible that a smouldering nephritis could have been present. This appears unlikely, as in each case the parents were quite definite that the child was well before the terminal illness; also the microscopic kidney changes are those of a recent event and not those of a smouldering nephritis. However, it is well known that hypertrophy of the cardiac muscle may appear quite quickly in children.

In the early stages of acute nephritis there is a considerable reduction in the glomerular filtration rate due to resistance in the glomerular arterioles (Black, Platt, Rowlands and Varley, 1948). This, combined with relatively normal tubular function, leads to sodium retention and consequently an increase in circulating blood volume. Cardiac enlargement, pulmonary oedema and cerebral oedema are all complications of sodium retention (Rosenheim, 1951). In view of the increase in circulating blood volume, one expects a rise in jugular venous pressure and the presence of peripheral oedema in these cases. In actual fact in neither of the first two cases was a raised jugular venous pressure recorded and peripheral oedema was carefully searched for but not found. In no case was the liver enlarged and the pulse rate was slow and regular in each case. The heart was not enlarged clinically and no abnormality was noticed on auscultation. Unfortunately, no biochemical tests were performed before death; nevertheless, these inconsistencies seem hard to reconcile with the sodium retention and increased plasma volume hypothesis.

Pulmonary oedema is known to occur in uraemia (Doniach, 1947), and the interstitial pulmonary oedema in the so-called uraemic lung was considered as an explanation in the first two cases of acute 
nephritis. In the first case the blood urea nitrogen was $22 \mathrm{mg} . / 100 \mathrm{ml}$. and in the second was $60 \mathrm{mg}$./ $100 \mathrm{ml}$. However, neither of these are high enough to invoke uraemia as the mechanism.

Another condition to be considered in the differential diagnosis in which renal and pulmonary lesions occur is polyarteritis with lung involvement as described by Rose and Spencer (1957). Nasal granulomata or granulomatous lesions in the respiratory tract were a feature (Wegener's granulomatosis). Peripheral arterial lesions and eosinophilia are also features of this disease. None of these were found in our cases and it was felt, therefore, that polyarteritis was unlikely as a diagnosis. Histopathological examination supported this clinical impression.

The roentgenological manifestations of pulmonary oedema have been described (Nessa and Rigler, 1941). They are non-specific as far as the aetiology is concerned and are subject to a wide range of variation. The physical findings are often minimal in the presence of extensive radiographic findings, so that a chest radiograph is invaluable in early diagnosis. The classical description of the chest radiograph is of butterfly-shaped symmetrical chest opacities with greatest density at the hilum and fading towards the periphery, leaving the apices and bases clear. The opacity has a homogeneous appearance and obliterates all lung detail. However, there are well-recognized variations in which the opacity has a fine irregular stippled appearance; it may be unilateral or even localized to one area of the lung, i.e. pneumonia complicated by pulmonary oedema.

The radiological appearances of the oedema of the lungs may be caused by many other disease processes. Similarly, the pathology described in these various situations may represent various stages of one causal process.

\section{Hypothesis}

It seems that none of the explanations offered is entirely satisfactory. At the risk of adding another, perhaps an aetiology based on an allergic mechanism has much to recommend it.

It appears that in each case the onset of the upper respiratory tract infection triggered off the fatal mechanism whatever it was. Case 1 had a cold one week before admission, Case 2 two days before admission, and Case 3 a pneumonia. The relation between a haemolytic streptococcal throat infection and development of acute nephritis is well known. It is thought that it is a reaction due to hypersensitivity to the infecting organisms. Therefore, it is reasonable to look for an explanation of the lung pathology along these lines also.
Although no haemolytic streptococci were isolated from the throats of either of the cases, in one brother of Case 2 the organisms were found. He had an upper respiratory tract infection at this time and was subsequently treated with penicillin.

It is tentatively suggested, therefore, that the haemolytic streptococcus may be the causal agent of the disease. The products of infection by this organism lead to the lesions of acute nephritis on the one hand and pulmonary oedema on the other. It is possible that there are all grades of this reaction, our first two cases representing the severe reaction with the accent on the lung, giving rise to rapidly fatal pulmonary oedema. Neither of these cases responded in any way to cortisone, but it was felt that the process was too well advanced for steroids to make any difference.

In the first case a penicillin allergy was considered in the diagnosis. It was thought, however, that the time relation and the type of reaction were unlike the reactions that are due to penicillin allergy. There were no skin lesions. No other allergens seemed to suggest themselves in either case, although Case 2 had a family history of allergy.

The pulmonary lesions in these cases bear a close resemblance to those described in rheumatic fever (Hadfield, 1938; Naish, 1928). This similarity perhaps supports a hypersensitivity reaction as the explanation, as also does the rapidity of onset of the reaction itself. As in rheumatic pneumonia, a relatively high proportion of the lung needs to be involved before symptoms appear. It is quite possible that the pathology is of much slower onset than appears clinically and only when the extent to which the lung is involved reaches a critical level do severe symptoms develop.

\section{Treatment}

Treatment in our three cases was disappointing. It has been suggested, many years ago, that positive pressure within the lungs would be of great assistance in the treatment of pulmonary oedema. This whole subject is well reviewed by Barach et al. (1938), who describe experimental work and the successful clinical use in eight cases of positive pressure respiration. They maintain that positive pressure within the alveoli decreases the amount of blood entering the right heart and in that way diminishes pulmonary congestion and facilitates the clearing of pulmonary oedema, and the direct opposing physical force on the external capillary wall tends to counteract any tendency to ooze serum.

In Case 2 positive pressure was tried as a method of treatment. It was not successful and presented 
practical difficulties in the conscious anoxic patient and is probably too late in the unconscious patient in any case. However, it offers the only positive approach that we have at present, and we are therefore persisting with its use.

As acute nephritis is a disease with a good prognosis in most cases, it appears that if some method were available for getting these children over this attack of pulmonary oedema the renal lesion would, in all probability, heal. It is hoped that this report may stimulate others to make further inquiries in this field in the hope of being able to find an answer to this problem.

The purpose of this report is to bring notice to bear on this condition of acute suffocative pulmonary oedema and to stress the importance of taking the blood pressure and examining the urine in any suspected case. Shortness of breath in any case following an upper respiratory tract infection is usually due to the onset of pneumonia. However, the knowledge that this condition begins in a similar fashion may help in early diagnosis.

\section{Summary}

Three fatal cases of acute nephritis leading to pulmonary oedema are described. An account of the pulmonary lesions is given and the possible mechanisms involved are discussed. The difficulties in diagnosis and treatment are stressed. A possible role of the haemolytic streptococcus in causing a sensitivity reaction affecting both lungs and kidneys is put forward as the most satisfactory explanation of the clinico-pathological findings. An increased awareness of the clinical condition may lead to early diagnosis and also to more work in this field which is long overdue and thence to some form of effective therapy for this distressing fatal condition.
I wish to thank the Board and Members of the Honorary Staff of the Adelaide Children's Hospital (Inc.) for permission to publish these cases. I am indebted to Dr. M. C. Fowler of the Pathology Department for the use of the pathological material, and to Mr. Ray Boyd for the preparation of the illustrations. I am grateful to Dr. M. Innis for his helpful comments in the preparation of the paper, and I wish to thank Miss U. Pridham for her help in typing this paper.

\section{REFERENCES}

Barach, Alvan L., Martin, John and Eckman, Morris (1938). Positive pressure respiration and its application to the treatment of adult pulmonary edema. Ann. intern. Med., 12, 754.

Black, D. A. K., Platt, R., Rowlands, E. N. and Varley. H. (1948). Renal haemodynamics in acute nephritis. Clin. Sci., 6, 295.

Brit. med. J. (1955). Annotation. Rheumatic pneumonia. 2, 1376. Cannon, P. R., Walsh, T. E. and Marshall, C. E. (1941). Acute local anaphylactic inflammation of the lungs. Amer. J. Path., 17, 777.

De Wardener, H.E. (1958). The Kidney. Churchill, London. Roentgenol., 58, 620.

French, A. J. (1946). Hypersensitivity in the pathogenesis of the histopathologic changes associated with sulphonamide chemotherapy. Amer. J. Path., 22, 679.

Goodpasture, E. W. (1919). The significance of certain pulmonary lesions in relation to the etiology of influenza. Amer. J. med. Sci., 158, 863 .

Granville-Grossman, K. L. and Sergeant, H. G. S. (1960). Pulmonary oedema due to salicylate intoxication. Lancet, 1, 575.

Hadfield, G. (1938). The rheumatic lung. Ibid., 2, 710.

- Holzel, A. and Fawcitt, J. (1960). Pulmonary changes in acute glomerulo-nephritis in childhood. J. Pediat., 57, 695.

Moutier, F. (1918). Hypertension et mort par oedème pulmonaire aigu. Presse méd., 26, 108.

Naish, A. E. (1928). The rheumatic lung. Lancet, 2, 10.

Nelson, Waldo E. (1959). Text Book of Pediatrics, 7th ed., p. 1038 Saunders, Philadelphia.

- Nessa, Curtis B. and Rigler, Leo G. (1941). The roentgenological manifestations of pulmonary edema. Radiology, 37, 35.

Ohlmacher, A. P. (1910). Acute pulmonary oedema as a terminal event in certain forms of epilepsy. Amer. J. med. Sci., 139, 417

Reid, J., Watson, R. D. and Sproull, D. H. (1950). The mode of action of salicylate in acute rheumatic fever. Quart. J. Med., 19, 1 .

Rose, G. A. and Spencer, H. (1957). Polyarteritis nodosa. Ibid., 26, 43.

Rosenheim, M. L. (1951). Sodium. Lancet, 2, 505.

Stanton, M. C. and Tange, J. D. (1958). Goodpasture's syndrome. Aust. Ann. Med., 7, 132 .

Sutcliffe, J. (1955). Pulmonary oedema due to salicylates with report of a case. Brit. J. Radiol., 28, 314

Weber, F. P. and Blum, K. (1942). Acute pulmonary oedema with hypoglycaemic coma. An example of acute pulmonary oedema of nervous origin. $J$. Neurol. Psychiat., 5,37 .

White, A. E. R. (1907). Clinical record of a case of acute suffocative pulmonary oedema. Intercolon. med. J. Aust., 12, 11.

Wood, P. (1956). Diseases of Heart and Circulation, 2nd ed., p. 638. Eyre and Spottiswoode, London. 